\title{
Treatment and prognosis after progression in long-term responders to EGFR-tyrosine kinase inhibitor in advanced non-small cell lung cancer
}

\author{
Zhengbo Song ${ }^{1,2}$, Yiping Zhang ${ }^{1,2}$
}

\begin{abstract}
1Department of Chemotherapy, Zhejiang Cancer Hospital, Hangzhou, China ${ }^{2}$ Key Laboratory Diagnosis and Treatment Technology on Thoracic Oncology, Zhejiang Province, Hangzhou, China
\end{abstract}

Submitted: 28 November 2013

Accepted: 13 February 2014

Arch Med Sci 2016; 12, 1: 107-111

DOI: 10.5114/aoms.2016.57586

Copyright @ 2016 Termedia \& Banach

\author{
Corresponding author: \\ Yiping Zhang MD \\ Department \\ of Chemotherapy \\ Zhejiang Cancer Hospital \\ 38 Guangji Road \\ 310022, Hangzhou, China \\ Phone: +8657188122182 \\ Fax: +8657188122188 \\ E-mail: zjzlyy16@163.com
}

\begin{abstract}
Introduction: The aim of this study was to investigate the treatment and prognosis of advanced non-small cell lung cancer (NSCLC) patients after failure of long-term treatment with epidermal growth factor receptor-tyrosine kinase inhibitor (EGFR-TKI).

Material and methods: We retrospectively analyzed all NSCLC patients with EGFR-TKI (gefitinib or erlotinib) treatment at our institution between 2011 and 2013 who progressed after at least stable disease on erlotinib or gefitinib for more than 6 months. Survival curves were plotted using the Kaplan-Meier method. The Cox proportional hazard model was used for multivariate analysis.

Results: In total, 521 patients were administered EGFR-TKI. Of these, 298 patients received EGFR-TKI with progression-free survival less than 6 months (group A), and the other 223 patients more than 6 months (group B). There was a significant difference in overall survival (OS) between group $A$ and group $B(7.2$ months vs. 5.0 months, $p<0.0001)$. The median OS for group B patients was 5.0 months. Among the 223 patients in group B, 38 patients received chemotherapy with continued EGFR-TKI after failure of prior gefitinib or erlotinib treatment, 92 with chemotherapy alone and 93 with best supportive care. Patients who continued gefitinib or erlotinib had a significantly longer OS (median: 7.5 months), followed by chemotherapy $(5.5$ months $)$ and best supportive care $(4.0$ months $)(p<0.001)$.

Conclusions: The prognosis of advanced NSCLC patients after failure of long-term treatment with EGFR-TKI was poor. Chemotherapy with continued EGFR-TKI beyond progression of long-term responders was feasible and led to prolonged OS in advanced NSCLC patients.
\end{abstract}

Key words: non-small cell lung cancer, EGFR-tyrosine kinase inhibitor, prognosis, efficacy.

\section{Introduction}

Lung cancer remains the leading cause of cancer mortality in the world [1]. Gefitinib, an oral small molecule agent that inhibits epidermal growth factor receptor (EGFR) tyrosine phosphorylation, is the first targeted agent to be approved for the treatment of advanced non-small cell lung cancer (NSCLC) patients, which demonstrated clinical efficacy in the second- or third-line treatment of NSCLC, especially in EGFR mutation 
patients [2, 3]. Erlotinib, another EGFR-tyrosine kinase inhibitor (EGFR-TKI), also has shown a survival benefit in second-line or third-line treatment for advanced NSCLC $[4,5]$.

Despite the high objective response rate (ORR) and disease control rate (DCR) in EGFR mutation patients with gefitinib or erlotinib treatment, most cases show disease progression. For patients who were previously treated with EGFR-TKI and later showed tumor progression, which is called EGFR TKI-acquired resistance, currently, many patients have no further treatment options [6-10]. The prognosis for this population is not well studied.

Conventional clinical practice at the time of tumor progression is to discontinue the EGFR-TKI and initiate a new chemotherapy or supportive treatment. Previous studies suggest that a heterogeneous tumor type exists at the time of acquired resistance, a subset of which remains sensitive to EGFR-TKI [11]. When the TKI is withdrawn from patients with acquired resistance, tumor growth may accelerate and then show efficacy when a TKI is re-administered $[12,13]$. As no prospective studies have focused on the treatment and prognosis after progression of EGFR-TKI, we do not know whether patients benefited from continued EGFR-TKI for NSCLC patients with EGFR-TKI acquired resistance.

In the present study, we investigated the treatment and prognosis of patients after failure of gefitinib or erlotinib in long-term responders to EGFR-TKI and focused on the efficacy of continued EGFR-TKI for NSCLC patients with acquired resistance.

\section{Material and methods}

\section{Patient eligibility}

Five hundred and twenty-one consecutive, unselected NSCLC patients, who were admitted to Zhejiang Cancer Hospital from March 2011 to May 2013, were administered with erlotinib or gefitinib treatment. NSCLC staging was performed for all the patients according to the $7^{\text {th }}$ TNM classification. Inclusion criteria were as follows: (1) pathologically proven primary stage IIIB or IV NSCLC, (2) all the patients benefited from the prior EGFR-TKI treatment with progression-free survival (PFS) more than 6 months, (3) disease recurrence or metastasis was confirmed using chest computed tomography (CT), brain magnetic resonance imaging (MRI) and bone scan as well as ultrasound examination and/or CT of the abdomen, (4) at least one measurable lesion and an Eastern Cooperative Oncology Group performance status of 0 to 3 .

\section{Follow-up}

All the patients were evaluated for tumor response and PFS. The follow-up rate was $100 \%$. The last follow-up date was Aug 31, 2013

\section{Statistical analysis}

The $\chi^{2}$ test was applied to evaluate the differences between different treatment arms. Overall survival (OS) was defined as the time from the first day of therapy to death, or until the date of the last follow-up visit for patients who were still alive. Survival analysis was conducted with a Kaplan-Meier analysis and log-rank test. A $p$-value of less than 0.05 was regarded as statistically significant. All statistical tests were analyzed using the computer software SPSS version 16.0 (SPSS Inc, Chicago, IL, USA).

\section{Results}

\section{Patient characteristics}

Overall, 521 patients were diagnosis for primary NSCLC and with EGFR-TKI therapy in due time of the treatment. Of these patients, 298 (57.2\%) patients had a PFS less than 6 months (group A) and 223 patients more than 6 months (group B). Among the 223 patients in group $B$, there were 102 males and 121 females. PS $0-1$ was present in 74 (33.2\%) patients and PS 2-3 accounted for $66.8 \%$. The median age of the patients was 55.0 years (range: 28-77 years). The predominant histology of the tumors was adenocarcinoma (187/223, 83.9\%). Forty-seven patients (47/223, $21.1 \%)$ had a smoking history. One hundred and thirty-eight patients were treated with gefitinib and 85 with erlotinib. Patients' characteristics in group $B$ are shown in Table I.

\section{Treatment after progression of EGFR-TKI and survival analysis in long-term responders to EGFR-TKI}

Of the 223 patients in Group B, 130 received treatment after progression of EGFR-TKI treatment, 92 received chemotherapy without continued EGFR-TKI, whereas 38 patients were treated with EGFR-TKI and chemotherapy beyond progression. Ninety-three patients received best supportive care.

The median OS in Group B was 27.5 months. The median OS from the failure of initial gefitinib or erlotinib treatment was 5.0 months $(95 \% \mathrm{Cl}$ : 4.5-5.6). Patients treated with continued EGFR-TKI had a significantly longer OS from progression of EGFR-TKI (median: 7.5 months), followed by chemotherapy (5.5 months). Patients with best supportive care had the worst prognosis (4.0 months) (Figure $1 ; p<0.001$ ).

\section{Efficacy comparison between chemotherapy and continued EGFR-TKI group in long-term responders to EGFR-TKI}

Partial responses were observed in 11 of 38 (28.9\%) patients in the continued EGFR-TKI group and in 10 of 92 (10.9\%) patients in the chemo- 
Table I. Clinical characteristics of 223 patients

\begin{tabular}{|c|c|}
\hline Parameter & Number (\%) \\
\hline \multicolumn{2}{|l|}{ Age: } \\
\hline Median & 55 \\
\hline$<65$ & $172(77.1)$ \\
\hline$\geq 65$ & $51(22.9)$ \\
\hline \multicolumn{2}{|l|}{ Gender: } \\
\hline Male & $102(45.7)$ \\
\hline Female & $121(54.3)$ \\
\hline \multicolumn{2}{|l|}{ Smoking history: } \\
\hline Current or ever & $47(21.1)$ \\
\hline Never & $176(77.9)$ \\
\hline \multicolumn{2}{|l|}{ Histology: } \\
\hline Adenocarcinoma & 187 (83.9) \\
\hline Non-adenocarcinoma & $36(26.1)$ \\
\hline \multicolumn{2}{|l|}{ EGFR-TKI: } \\
\hline Gefitinib & $138(61.9)$ \\
\hline Erlotinib & $85(38.1)$ \\
\hline \multicolumn{2}{|l|}{ PS: } \\
\hline $0-1$ & $74(33.2)$ \\
\hline $2-3$ & $149(66.8)$ \\
\hline \multicolumn{2}{|l|}{ Stage: } \\
\hline IIIB & $15(6.7)$ \\
\hline IV & $208(92.3)$ \\
\hline \multicolumn{2}{|l|}{ TKI as which line: } \\
\hline First-line & $55(24.7)$ \\
\hline Second or further-line & $168(75.3)$ \\
\hline \multicolumn{2}{|l|}{ Continued EGFR-TKI: } \\
\hline Yes & $38(17.0)$ \\
\hline No & $185(83.0)$ \\
\hline
\end{tabular}

therapy alone group $(p=0.02)$. Median PFS was longer in the continued EGFR-TKI group than the chemotherapy alone group (4.0 months vs. 2.8 months, $p=0.04)$. There was a significant difference in OS between the continued EGFR-TKI group and the chemotherapy group (7.5 months vs. 5.0 months, $p=0.008$ ).

\section{Prognosis and progression modes in long-term responders to EGFR-TKI}

There were significant difference in OS between short-term and long-term responders to

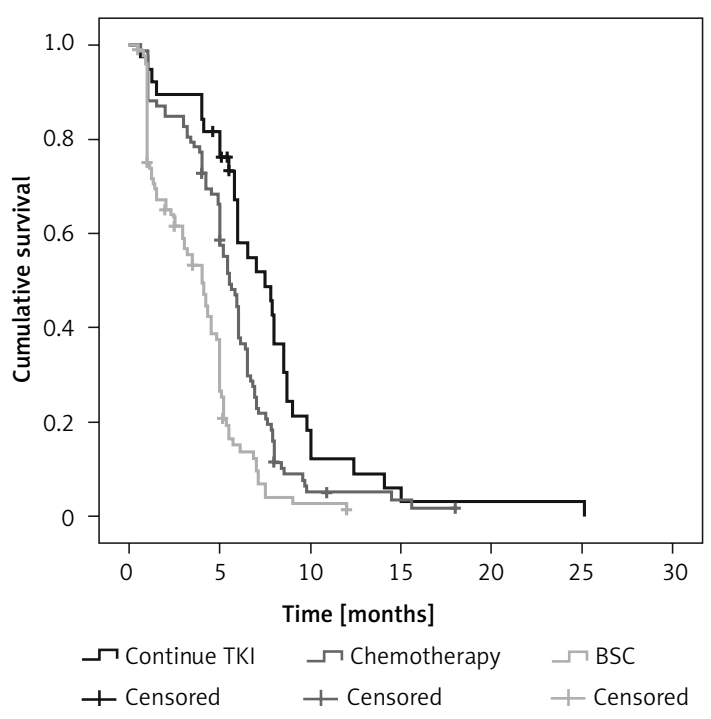

Figure 1. Overall survival of EGFR-TKI in addition to chemotherapy, chemotherapy alone and best supportive treatment $(p<0.001)$

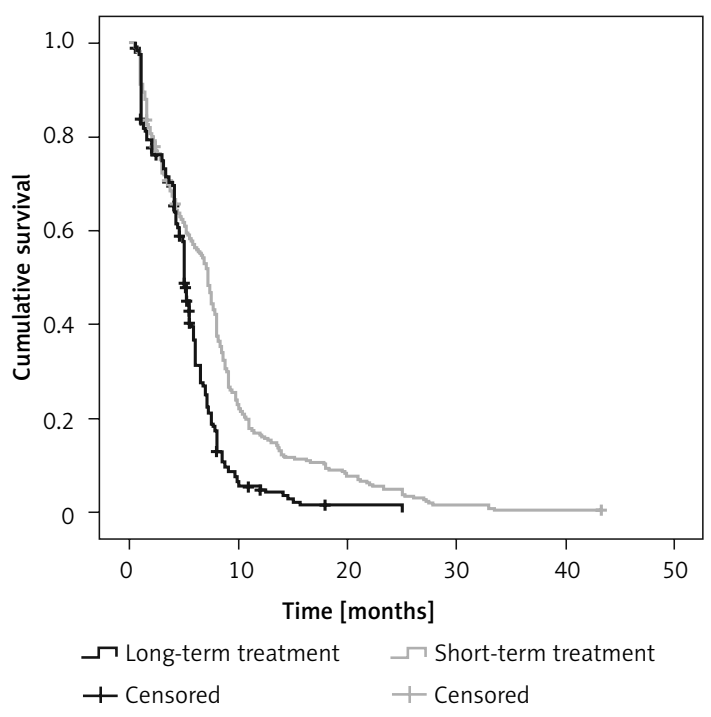

Figure 2. Comparison of overall survival in patients between short-term and long-term responders to EGFR-TKI $(p<0.001)$

EGFR-TKI (7.2 months vs. 5.0 months, $p<0.0001)$ (Figure 2).

According to Yang et al. [14], among clinical modes of EGFR-TKI failure, 126 cases were classified as dramatic progression (56.5\%), 42 as gradual progression (18.8\%) and 55 as local progression $(24.7 \%)$ in group B. There were significant difference in OS among different models in group $B$ (Figure 3, $p=0.001$ ). The median OS of the gradual progression group (6.7 months) was longer than that of the dramatic progression group (4.0 months; $p<0.001$ ). No significant difference was found regarding the median OS between gradual and local progression groups (6.7 months vs. 5.0 months; $p=0.067$ ). 


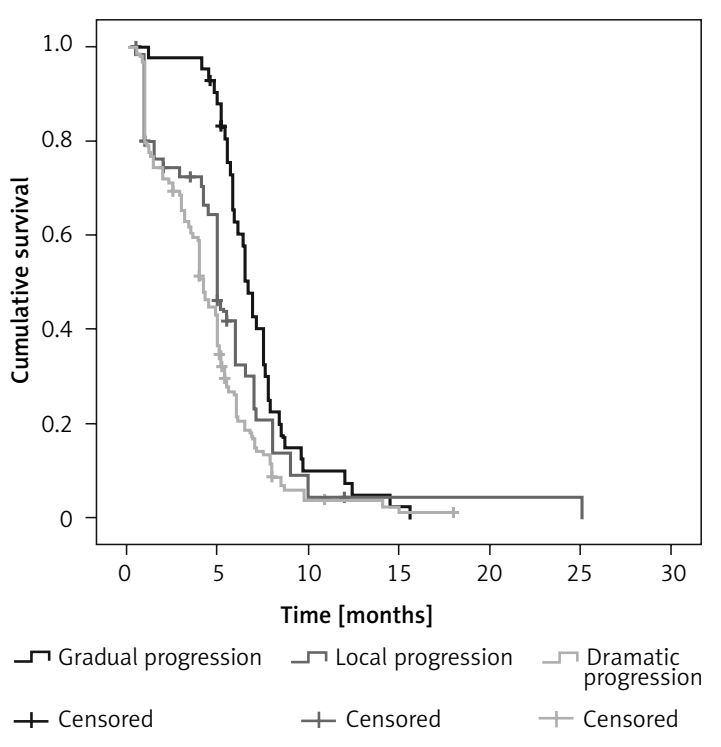

Figure 3. Overall survival in different progression groups $(p=0.001)$

Table II. Univariate predictors of OS in 223 patients

\begin{tabular}{|lccc|}
\hline Variables & OS & $95 \% \mathrm{Cl}$ & $P$-value \\
\hline Age: & & & 0.143 \\
\hline$<65$ & 5 & $4.3-5.9$ & \\
\hline$\geq 65$ & 5.6 & $4.5-6.1$ & \\
\hline \begin{tabular}{l} 
Gender: \\
\hline Male
\end{tabular} & 5 & $4.8-5.2$ & \\
\hline Female & 5.2 & $4.8-5.5$ & \\
\hline Smoking history: & & & 0.11 \\
\hline Current or ever & 5 & $4.8-5.2$ & \\
\hline Never & 5.1 & $4.4-5.7$ & \\
\hline Histology: & & & 0.172 \\
\hline Adenocarcinoma & 5.5 & $4.5-5.9$ & \\
\hline Non-adenocarcinoma & 4.6 & $4.4-5.6$ & \\
\hline EGFR-TKI: & & & 0.213 \\
\hline Gefitinib & 4.8 & $4.5-5.5$ & \\
\hline Erlotinib & 5.2 & $4.2-6.0$ & \\
\hline PS: & & & $<0.001$ \\
\hline O-1 & 7 & $5.9-8.0$ & \\
\hline 2-3 & 4.2 & $3.5-4.9$ & \\
\hline Continued TKI: & & & $<0.001$ \\
\hline Yes & 7.5 & $5.4-9.6$ & \\
\hline No & 5 & $4.6-5.4$ & \\
\hline TKI as which line: & & & 0.116 \\
\hline First-line & 5.6 & $4.4-6.0$ & \\
\hline Second or further-line & 4.6 & $4.4-5.6$ & \\
\hline Stage: & & & 0.756 \\
\hline IIIB & 5.2 & $4.6-5.5$ & \\
\hline IV & $5.4-5.2$ & \\
\hline
\end{tabular}

Factors affecting OS in univariate and multivariate analysis

Results of univariate analysis for OS of group B are shown in Table II. The performance status score (PS) and continued EGFR-TKI treatment were the factors influencing the OS. No other factors correlated significantly with OS.

Among the 223 patients, 87 patients provided tumor samples for EGFR mutation analysis. EGFR mutations were identified in 78 patients, 7 with EGFR wild-type. For the 87 patients with EGFR mutation status identified, there was no difference in the OS between the EGFR mutation and wild-type patients (5.6 months vs. 5.0 months, $p=0.56$ ).

A multivariate Cox regression model was constructed with the incorporation of age, gender, stage, PS, smoking history, and continued EGFRTKI treatment. Performance status and continued EGFR-TKI treatment remained as independent prognostic factors for OS (Table III).

\section{Discussion}

The introduction of EGFR-TKI has notably expanded the available therapeutic options for patients with advanced NSCLC. However, there was no standard treatment for these patients after progression, and the prognosis remains unclear [7-10]. Our data show that the prognosis is poor and treatment with continued EGFR-TKI beyond progression in addition to chemotherapy is feasible in long-term EGFR-TKI responders. To the best of our knowledge, this is the largest report that has focused on the prognosis and treatment strategy in patients with failure of long-term treatment with EGFR-TKI.

The prognosis for patients who benefited from EGFR-TKI is not well studied. The OS is longer in group A than group B (Figure 2), which might be due to the fact that $41.7 \%$ of patients in group $B$ did not receive further-line therapy. Another rea-

Table III. Multivariate predictors of overall survival in 223 patients

\begin{tabular}{|lccc|}
\hline Variables & HR & $95 \% \mathrm{Cl}$ & $P$-value \\
\hline Age & 1.024 & $0.780-1.344$ & 0.866 \\
\hline Gender & 0.785 & $0.556-1.109$ & 0.169 \\
\hline Smoking history & 1.192 & $0.896-1.586$ & 0.227 \\
\hline Histology & 0.998 & $0.759-1.311$ & 0.986 \\
\hline TKI type & 0.824 & $0.607-1.118$ & 0.214 \\
\hline PS & 2.643 & $1.885-3.708$ & $<0.001$ \\
\hline $\begin{array}{l}\text { Continued } \\
\text { EGFR-TKI }\end{array}$ & 2.085 & $1.375-3.161$ & 0.001 \\
\hline Stage & 1.200 & $0.906-1.589$ & 0.203 \\
\hline
\end{tabular}


son may due to more than half of patients in group B classified as dramatic progression. Established treatment modes after EGFR-TKI failure are lacking nowadays. A clinical treatment model was determined based on clinical observations in the retrospective study by Yang et al. [14]. The progression type was divided into three modes based on the duration of disease control and evolution of tumor burden, which were dramatic progression, gradual progression, and local progression. According to the Yang et al. model, there were significant difference in OS between different models in our study (Figure 2).

In patients with EGFR-mutant lung cancer and acquired resistance to EGFR-TKI, discontinuation of EGFR-TKI before initiation of new treatment is associated with a clinically significant risk of accelerated disease progression in the study by Chaft et al. [6]. Continuation of EGFR-TKI with chemotherapy beyond progression may be a good choice for patients with acquired resistance. There was a significantly longer OS from progression on EGFR-TKI for the patients who continued gefitinib or erlotinib and chemotherapy than chemotherapy alone in the Faehling et al. study [15]. A retrospective study by Goldberg et al. showed that continuation of EGFR-TKI with chemotherapy in patients with acquired resistance improves the response rate but not PFS and OS compared with chemotherapy alone [16]. Unlike the Goldberg et al. study, continuation of EGFR-TKI with chemotherapy increased the response rate and OS in our study. However, due to the small group size of the previous two studies (25 patients in the Faehling et al. study and 28 in the Goldberg et al. study) and our study (38 patients), this work must be further prospectively validated in population-based research.

The present study possesses intrinsic limitations due to its retrospective design. In addition, less than half of our patients received EGFR mutation information, which also influenced our clinical analysis. However, as the largest number of data, our retrospective study can also be considered to be meaningful.

In conclusion, our data suggest that the prognosis is poor in patients who progressed from long-term EGFR-TKI treatment. Continuing EGFRTKI beyond initial progression in addition to chemotherapy is feasible and effective.

\section{Acknowledgments}

This work was supported by a grant from the Foundation of Zhejiang Province (No. 2013KYB049).

\section{Conflict of interest}

The authors declare no conflict of interest.
References

1. Siegel R, Ward E, Brawley O, Jemal A. Cancer statistics 2011. CA Cancer J Clin 2011; 61: 212-36.

2. Maruyama R, Nishiwaki Y, Tamura T, et al. Phase III study, V-15-32, of gefitinib versus docetaxel in previously treated Japanese patients with non-small-cell lung cancer. J Clin Oncol 2008; 26: 4244-52.

3. Thatcher N, Chang A, Parikh P, et al. Gefitinib plus best supportive care in previously treated patients with refractory advanced non-small-cell lung cancer: results from a randomised, placebo-controlled, multicentre study (Iressa Survival Evaluation in Lung Cancer). Lancet 2005; 366: 1527-37.

4. Ciuleanu T, Stelmakh L, Cicenas S, et al. Efficacy and safety of erlotinib versus chemotherapy in second-line treatment of patients with advanced, non-small-cell lung cancer with poor prognosis (TITAN): a randomised multicentre, open-label, phase 3 study. Lancet Oncol 2012; 13: 300-8.

5. Shepherd FA, Rodrigues Pereira J, Ciuleanu T, et al. Erlotinib in previously treated non-small-cell lung cancer. N Engl J Med 2005; 353: 123-32.

6. Chaft JE, Oxnard GR, Sima CS, et al. Disease flare after tyrosine inhibitor discontinuation in patients with erlotinib or gefitinib: implications for clinical trial design. Clin Cancer Res 2011; 17: 6298-303.

7. Song Z, Zhang Y. Efficacy of gefitinib or erlotinib in patients with squamous cell lung cancer. Arch Med Sci 2015; 11: 164-8.

8. Oxnard GR. Strategies for overcoming acquired resistance to epidermal growth factor receptor: targeted therapies in lungcancer. Arch Pathol Lab Med 2012; 136: 1205-9.

9. Kuo CH, Lin SM, Lee KY, et al. Subsequent chemotherapy improves survival outcome in advanced non-small-cell lung cancer with acquired tyrosine kinase inhibitor resistance. Clin Lung Cancer 2010; 11: 51-6.

10. Lee JC, Jang SH, Lee KY, et al. Treatment of non-small cell lung carcinoma after failure of epidermal growth factor receptor tyrosine kinase ihibitor. Cancer Res Treat 2013; 45: 79-85.

11. Chmielecki J, Foo J, Oxnard GR, et al. Optimization of dosing for EGFR-mutant non-small cell lung cancer with evolutionary cancer modeling. Sci Transl Med 2011; 3: 90ra59.

12. Song ZB, Yu YF, Chen ZW, Lu S. Erlotinib as a salvage treatment for advanced non-small-cell lung cancer patients after failure of gefitinib treatment. Chin Med J (Engl) 2011; 124: 2279-83.

13. Song Z, He C, Zhang B, Zhang Y. Re-administration after the failure of gefitinib or erlotinib in patients with advanced non-small cell lung cancer. J Thorac Dis 2013; 5: 400-5.

14. Yang JJ, Chen HJ, Yan HH, et al. Clinical modes of EGFR tyrosine kinase inhibitor failure and subsequent management in advanced non-small cell lung cancer. Lung Cancer 2013; 79: 33-9.

15. Faehling M, Eckert R, Kamp T, et al. EGFR-tyrosine kinase inhibitor treatment beyond progression in long-term Caucasian responders to erlotinib in advanced nonsmall cell lung cancer: a case-control study of overall survival. Lung Cancer 2013; 80: 306-12.

16. Goldberg SB, Oxnard GR, Digumarthy S, et al. Chemotherapy with erlotinib or chemotherapy alone in advanced non-small cell lung cancer with acquired resistance to EGFR tyrosine kinase inhibitors. Oncologist 2013; 18: 1214-20. 